\title{
Modular reduction in abstract polytopes
}

\author{
B. Monson* and Egon Schulte ${ }^{\dagger}$
}

November 9, 2018

\begin{abstract}
The paper studies modular reduction techniques for abstract regular and chiral polytopes, with two purposes in mind: first, to survey the literature about modular reduction in polytopes; and second, to apply modular reduction, with moduli given by primes in $\mathbb{Z}[\tau]$ (with $\tau$ the golden ratio), to construct new regular 4-polytopes of hyperbolic types $\{3,5,3\}$ and $\{5,3,5\}$ with automorphism groups given by finite orthogonal groups.
\end{abstract}

Key Words: abstract polytopes, regular and chiral, Coxeter groups, modular reduction AMS Subject Classification (2000): Primary: 51M20. Secondary: 20F55.

\section{Introduction}

Polytopes and their symmetry have inspired mathematicians since antiquity. In the past three decades, a modern abstract theory of polytopes has emerged featuring an attractive interplay of mathematical areas, including geometry, combinatorics, group theory, graph theory and topology (see [21]). Abstract polytopes share many properties with ordinary convex polytopes but a priori are not embedded in the geometry of an ambient space.

The present paper studies modular reduction techniques for regular and chiral abstract polytopes. Modular reduction has proved to be a powerful tool in the construction and analysis of new classes of polytopes. Our paper serves two purposes: first, to survey the literature about modular reduction techniques in polytopes; and second, to apply modular reduction, with moduli given by primes in the ring of integers $\mathbb{Z}[\tau]$ of the quadratic number field $\mathbb{Q}(\sqrt{5})$, to construct new infinite classes of regular 4-polytopes of hyperbolic types $\{3,5,3\}$ and $\{5,3,5\}$ with automorphism groups given by orthogonal groups over finite fields.

Last, but not least, as a birthday greeting, we wish to acknowledge the many contributions by our friend and colleague Ted Bisztriczky in convexity, polytope theory and combinatorial geometry (see also [2]).

\footnotetext{
*Supported by NSERC of Canada Grant \# 4818

${ }^{\dagger}$ Supported by NSA-grant H98230-07-1-0005
} 


\section{Basic notions and methods}

For general background material on abstract polytopes we refer the reader to [21, Chs. 2,3]. Here we just review some basic terminology.

An (abstract) polytope of rank $n$, or an $n$-polytope, is a partially ordered set $\mathcal{P}$ with a strictly monotone rank function having range $\{-1,0, \ldots, n\}$. An element of rank $j$ is called a $j$-face of $\mathcal{P}$, and a face of rank 0,1 or $n-1$ is a vertex, edge or facet, respectively. The maximal chains, or flags, of $\mathcal{P}$ all contain exactly $n+2$ faces, including a unique least face $F_{-1}$ (of rank -1 ) and a unique greatest face $F_{n}$ (of rank $n$ ). Two flags are said to be adjacent ( $i$-adjacent) if they differ in a single face (just their $i$-face, respectively). Then $\mathcal{P}$ is required to be strongly flag-connected (see [21, Ch.2]). Finally, $\mathcal{P}$ has the following homogeneity property: whenever $F \leq G$, with $F$ a $(j-1)$-face and $G$ a $(j+1)$-face for some $j$, then there are exactly two $j$-faces $H$ with $F \leq H \leq G$.

Whenever $F \leq G$ are faces of ranks $j \leq k$ in $\mathcal{P}$, the section $G / F:=\{H \in \mathcal{P} \mid F \leq H \leq$ $G\}$ is thus a $(k-j-1)$-polytope in its own right. In particular, we can identify $F$ with $F / F_{-1}$. Moreover, we call $F_{n} / F$ the co-face at $F$, or the vertex-figure at $F$ if $F$ is a vertex.

Our interest is primarily in regular or chiral polytopes. A polytope $\mathcal{P}$ is regular if its automorphism group $\Gamma(\mathcal{P})$ is transitive on the flags of $\mathcal{P}$, and $\mathcal{P}$ is chiral if $\Gamma(\mathcal{P})$ has two flag orbits such that adjacent flags are always in distinct orbits.

For a regular polytope $\mathcal{P}$, the group $\Gamma(\mathcal{P})$ is generated by $n$ involutions $\rho_{0}, \ldots, \rho_{n-1}$, where $\rho_{i}$ maps a fixed, or base, flag $\Phi$ to the flag $\Phi^{i}, i$-adjacent to $\Phi$. These generators satisfy (at least) the standard Coxeter-type relations

$$
\left(\rho_{i} \rho_{j}\right)^{p_{i j}}=\epsilon \quad \text { for } i, j=0, \ldots, n-1
$$

where $p_{i i}=1, p_{j i}=p_{i j}=: p_{i+1}$ if $j=i+1$, and $p_{i j}=2$ otherwise; thus the underlying Coxeter diagram is a string diagram. Note that $p_{j}:=p_{j-1, j} \in\{2,3, \ldots, \infty\}$ for $j=1, \ldots, n-1$. These numbers determine the $(S c h l a ̈ f l i)$ type $\left\{p_{1}, \ldots, p_{n-1}\right\}$ of $\mathcal{P}$. Moreover, the following intersection condition holds:

$$
\left\langle\rho_{i} \mid i \in I\right\rangle \cap\left\langle\rho_{i} \mid i \in J\right\rangle=\left\langle\rho_{i} \mid i \in I \cap J\right\rangle \quad(I, J \subseteq\{0,1, \ldots, n-1\}) .
$$

The rotations $\sigma_{i}:=\rho_{i} \rho_{i-1}(i=1, \ldots, n-1)$ generate the rotation subgroup $\Gamma^{+}(\mathcal{P})$ of $\Gamma(\mathcal{P})$, which is of index at most 2 . We call $\mathcal{P}$ directly regular if this index is 2 .

A group $\Gamma=\left\langle\rho_{0}, \ldots, \rho_{n-1}\right\rangle$ whose generators satisfy (11) and (2), is called a string $C$ group; here, the "C" stands for "Coxeter", though not every C-group is a Coxeter group. These string C-groups are precisely the automorphism groups of regular polytopes, since, in a natural way, such a polytope can be uniquely reconstructed from $\Gamma$ (see [21, $\S 2 \mathrm{E}]$ ). Therefore, we often identify a regular polytope with its automorphism (string C-) group.

The group $\Gamma(\mathcal{P})$ of a chiral polytope $\mathcal{P}$ is generated by $n-1$ elements $\sigma_{1}, \ldots, \sigma_{n-1}$, which again are associated with a base flag $\Phi=\left\{F_{1}, F_{0}, \ldots, F_{n}\right\}$, such that $\sigma_{i}$ fixes all the faces in $\Phi \backslash\left\{F_{i-1}, F_{i}\right\}$ and cyclically permutes ("rotates") consecutive $i$-faces of $P$ in the (polygonal) section $F_{i+1} / F_{i-2}$ of rank 2. By replacing a generator by its inverse if need be, we can further require that, if $F_{i}^{\prime}$ denotes the $i$-face of $\mathcal{P}$ with $F_{i-1}<F_{i}^{\prime}<F_{i+1}$ and $F_{i}^{\prime} \neq F_{i}$, then $\sigma_{i}\left(F_{i}^{\prime}\right)=F_{i}$. The resulting distinguished generators $\sigma_{1}, \ldots, \sigma_{n-1}$ of $\Gamma(\mathcal{P})$ then satisfy 
relations

$$
\sigma_{i}^{p_{i}}=\left(\sigma_{j} \sigma_{j+1} \ldots \sigma_{k}\right)^{2}=\epsilon \text { for } i, j, k=1, \ldots, n-1, \text { with } j<k,
$$

where again the numbers $p_{i}$ determine the type $\left\{p_{1}, \ldots, p_{n-1}\right\}$ of $P$. Moreover, $\Gamma(\mathcal{P})$ and its generators satisfy a certain intersection condition resembling that for C-groups. Conversely, if $\Gamma$ is a group generated by $\sigma_{1}, \ldots, \sigma_{n-1}$ such that the relations (3) and the new intersection condition hold, then $\Gamma$ is the group of a chiral polytope, or the rotation subgroup for a directly regular polytope; here the polytope is regular if and only if $\Gamma$ admits an involutory automorphism $\rho$ such that $\rho\left(\sigma_{1}\right)=\sigma_{1}^{-1}, \rho\left(\sigma_{2}\right)=\sigma_{1}^{2} \sigma_{2}$, and $\rho\left(\sigma_{j}\right)=\sigma_{j}$ for $j \geq 2$. For a chiral polytope $\mathcal{P}$, the two flag orbits yield two sets of generators $\sigma_{i}$ which are not conjugate in $\Gamma(\mathcal{P})$; thus a chiral polytope occurs in two enantiomorphic (mirror image) forms.

We now describe the basic idea of modular reduction. We begin with a linear group $G$ over a ring $\mathbb{D}$, choose an ideal $J$ of $\mathbb{D}$, and try to construct a polytope from the quotient group of $G$ obtained by viewing $G$ as a linear group over $\mathbb{D} / J$. For the latter step the main obstruction is typically the intersection condition for the resulting quotient group of $G$. In most applications, $G$ itself is already a string C-group (often a Coxeter group), $\mathbb{D}$ is the ring of integers in an algebraic number field, and $J$ is an ideal of $\mathbb{D}$.

More precisely, let $\mathbb{D}$ be a commutative ring with identity 1 , let $V$ be a free module over $\mathbb{D}$ of rank $n$ with basis $b_{0}, \ldots, b_{n-1}$, and let $G$ be a subgroup of the general linear group $G L_{n}(\mathbb{D})$ over $\mathbb{D}$, whose elements we may view as invertible linear transformations (module isomorphisms) of $V$. Now let $J$ be an ideal of $\mathbb{D}$. Then the natural ring epimorphism $\mathbb{D} \rightarrow \mathbb{D} / J$ defined by $a \rightarrow a+J$ induces a group epimorphism $G \rightarrow G^{J}$, where $G^{J}$ is a subgroup of $G L_{n}(\mathbb{D} / J)$, the modular reduction of $G$; in other words, we obtain $G^{J}$ by simply viewing the matrix entries of the elements in $G$ as entries in $\mathbb{D} / J$. Then $G^{J}$ naturally acts on the free module $V^{J}$ over $\mathbb{D} / J$ of rank $n$ with basis $b_{0}^{J}, \ldots, b_{n-1}^{J}$. We often abuse notation by referring to the modular images of objects by the same name (such as $V$ or $b_{0}, \ldots, b_{n-1}$, etc.); that is, we drop the superscript $J$.

Modular reduction is, of course, a natural idea and has been used in various ways to construct maps and polytopes [28, 29, 35]. Here we begin by applying the idea to crystallographic Coxeter groups.

\section{Polytopes from crystallographic Coxeter groups}

Let $\Gamma$ be an abstract string Coxeter group with generators $\rho_{0}, \ldots, \rho_{n-1}$ and presentation as in (11), where again $p_{i i}=1$ and $p_{i j}=2$ for $|i-j| \geq 2$. Let $V$ be real $n$-space, with basis $\alpha=\left\{a_{0}, \ldots, a_{n-1}\right\}$ and symmetric bilinear form $x \cdot y$ defined by

$$
a_{i} \cdot a_{j}:=-2 \cos \frac{\pi}{p_{i j}}, \quad 0 \leq i, j \leq n-1
$$

Let $R: \Gamma \rightarrow G$ be the (faithful) standard representation of $\Gamma$ in $V$, where $G=\left\langle r_{0}, \ldots, r_{n-1}\right\rangle$ is the isometric reflection group on $V$ generated by the reflections with roots $a_{i}$ (see [14, $\S 5.3-5.4])$; thus,

$$
r_{i}(x)=x-\left(x \cdot a_{i}\right) a_{i} \quad(i=0, \ldots, n-1) .
$$

Now let $m=2 m^{\prime}$, where $m^{\prime}$ is the lowest common multiple of all $p_{i j}$ which are finite. Let $\xi$ be a primitive $m$-th root of unity, and let $\mathbb{D}:=\mathbb{Z}[\xi]$. Then, with respect to the basis $\alpha$ of $V$, the 
reflections $r_{j}$ are represented by matrices in $G L_{n}(\mathbb{D})$ so that we may view $G$ as a subgroup of $G L_{n}(\mathbb{D})$. (By [9, Th. 21.13], $\mathbb{D}$ is the ring of integers in the algebraic number field $\mathbb{Q}(\xi)$; and $\mathbb{D}$ has (finite) rank $\phi(m)$ as a $\mathbb{Z}$-module.) Now we can reduce $G \bmod p$, for any prime $p$, here allowing $p=2[9$, ch. XII]. More precisely, suppose that $p$ is a rational prime and that $J$ is a maximal ideal in $\mathbb{D}$ with $p \mathbb{D} \subseteq J \subset \mathbb{D}$. Then $\mathbb{K}:=\mathbb{D} / J$ is a finite field of characteristic $p$, and reduction mod $p$ of $G$ is achieved by applying the natural epimorphism $\mathbb{D} \rightarrow \mathbb{K}$ to the matrix entries of the elements in $G$. This then defines a representation $\kappa: G \rightarrow G L_{n}(\mathbb{K})$ with image group $G^{p}:=G^{J}=\kappa(G)$. (This construction is essentially independent of the choice of $J$.) Note that $\kappa$ is faithful when $G$ is finite and $p \nmid|G|$ (in fact, $\kappa$ often is faithful even when $p$ divides $|G|)$. In any case, $\operatorname{ker} \kappa$ is a $p$-subgroup of $G$.

The modular reduction technique is considerably more straightforward for crystallographic Coxeter groups, meaning that $G$ (or $\Gamma$ ) leaves invariant some lattice in $V$. A (string) Coxeter group $G$ is known to be crystallographic if and only if $p_{i j}=2,3,4,6$ or $\infty$ for all $i \neq j$. In fact, even allowing non-string diagrams, it is also true that $G$ is crystallographic if and only if there is a basic system $\beta=\left\{b_{0}, \ldots, b_{n-1}\right\}$, with $b_{i}:=t_{i} a_{i}$ for certain $t_{i}>0$, such that $m_{i j}:=-t_{i}^{-1}\left(a_{i} \cdot a_{j}\right) t_{j} \in \mathbb{Z}$ for $0 \leq i, j \leq n-1$. Then, for the rescaled roots $b_{i}$, we have

$$
r_{i}\left(b_{j}\right)=b_{j}+m_{i j} b_{i}
$$

so that the generators $r_{i}$ are represented by integral matrices with respect to the basis $\beta$, and the corresponding root lattice $\oplus_{j} \mathbb{Z} b_{j}$ actually is $G$-invariant. Thus we may take $\mathbb{D}=\mathbb{Z}$, and $G$ can be reduced modulo any integer $d \geq 2$. We focus primarily on the case when $p$ is an odd prime, but address some questions regarding composite moduli as well. For a composite modulus $d \geq 2$, the reduced group $G^{d}$ generally does not "split" according to the prime factorization of $d$, and its structure depends more heavily on the diagram $\Delta(G)$ used in the reduction process (two diagrams which modulo a prime are equivalent, may not be equivalent modulo a composite modulus). We now describe these diagrams.

The various possible basic systems $\left\{t_{i} a_{i}\right\}$ for a crystallographic Coxeter group $G$ can be represented by a diagram $\Delta(G)$ (see [5, p. 415] or [22]): for $0 \leq i, j \leq n-1$, node $i$ is labelled $2 t_{i}^{2}\left(=b_{i}^{2}\right)$; and distinct nodes $i \neq j$ are joined by $\lambda_{i j}:=\min \left\{m_{i j}, m_{j i}\right\}$ unlabelled branches. Note that $\lambda_{i j}=\lambda_{j i}=0,1$ or 2 , so the underlying graph is essentially that of the underlying Coxeter diagram $\Delta_{c}(G)$ of $G$, except that a mark $p_{i j}=\infty$ is indicated by a doubled branch in the case that $m_{i j}=m_{j i}=2$. In Table 1 we display the possible subdiagrams corresponding to the dihedral subgroups $\left\langle r_{i}, r_{j}\right\rangle$. For simplicity we have replaced the node labels $2 t_{i}^{2}, 2 t_{j}^{2}$ by $s, t$ or $s, k s(k=1,2,3,4)$, as appropriate. Note here that $m_{i j} m_{j i}=4 \cos ^{2} \frac{\pi}{p_{i j}}$, so that, if $m_{i j} \geq m_{j i} \geq 1$, we have $t_{j}^{2} / t_{i}^{2}=m_{i j} / m_{j i}=1,2,3,4$ (or 1 ) for $p_{i j}=3,4,6, \infty$, respectively.

\begin{tabular}{|c|c|c|}
\hline Nodes $i, j$ & Parameters & $\lambda_{i j}$ \\
\hline $\begin{array}{ll}s & t \\
\bullet & \bullet \\
\end{array}$ & $p_{i j}=2$ & 0 \\
\hline $\begin{array}{ll}s & k s \\
\bullet & \bullet\end{array}$ & $\begin{aligned} p_{i j} & =3,4,6, \infty \\
(k & =1,2,3,4)\end{aligned}$ & 1 \\
\hline$\stackrel{s}{\bullet} \bullet$ & $\begin{array}{c}p_{i j}=\infty \\
(k=1)\end{array}$ & 2 \\
\hline
\end{tabular}

Table 1. Basic Systems for the Crystallographic Dihedral Groups $\left\langle r_{i}, r_{j}\right\rangle$. 
The Gram matrix $B=\left[b_{i j}\right]:=\left[b_{i} \cdot b_{j}\right]$ is easily computed from $\Delta(G)$, since $b_{i i}=2 t_{i}^{2}$ is simply the label attached to node $i$, and $b_{i j}=-\lambda_{i j} \max \left\{b_{i i}, b_{j j}\right\} / 2$ for $i \neq j$. Moreover, $m_{i j}=\lambda_{i j} \max \left\{1, b_{j j} / b_{i i}\right\}$ for $i \neq j$.

For a connected Coxeter diagram $\Delta_{c}(G)$, the corresponding group $G$ has to scale only finitely many basic systems $\beta$. Any such system is represented by an essentially unique diagram $\Delta(G)$, in which node labels form a set of relatively prime integers. For two such basic systems $\beta, \beta^{\prime}$, we can convert from $\Delta(G)$ to $\Delta^{\prime}(G)$ by consecutively adjusting the labels and branches of various pairs of adjacent nodes by operations of the following kind: inverting the ratio of the node labels; doubling a single branch and balancing its labels, or converting a double branch to a single branch, with ratio 4 , if the corresponding branch in $\Delta_{c}(G)$ is marked $\infty$. Following these adjustments on pairs of nodes, we may finally have to rescale the entire set of labels to obtain a set of relatively prime integers.

Then we may reduce $G$ modulo an odd prime $p$ to obtain a subgroup $G^{p}$ of $G L_{n}\left(\mathbb{Z}_{p}\right)$ generated by the modular images of the $r_{i}$ 's. We call the prime $p$ generic for $G$ if $p \geq 5$, or $p=3$ but no branch of $\Delta_{c}(G)$ is marked 6. In the generic case, no node label of $\Delta(G)$ vanishes mod $p$ and a change in the underlying basic system for $G$ has the effect of merely conjugating $G^{p}$ in $G L_{n}\left(\mathbb{Z}_{p}\right)$. On the other hand, in the non-generic case, the group $G^{p}$ may depend essentially on the actual diagram $\Delta(G)$ used in the reduction mod $p$. For any odd prime $p$, we always find that $G^{p}=\left\langle r_{0}, \ldots, r_{n-1}\right\rangle$ is a subgroup of the orthogonal group $O\left(\mathbb{Z}_{p}^{n}\right)$ of isometries for the (possibly singular) symmetric bilinear form $x \cdot y$, the latter being defined on $\mathbb{Z}_{p}^{n}$ by means of (the modular image of) the Gram matrix $B$; in particular, $r_{i}$ is the orthogonal reflection with root $b_{i}$ if $b_{i}^{2} \neq 0$.

Now recall from [23, Thm. 3.1] that an irreducible group $G^{p}$ of the above sort, generated by $n \geq 3$ reflections, must necessarily be one of the following:

- an orthogonal group $O(n, p, \epsilon)=O(V)$ or $O_{j}(n, p, \epsilon)=O_{j}(V)$, excluding the cases $O_{1}(3,3,0), O_{2}(3,5,0), O_{2}(5,3,0)$ (supposing for these three that $\operatorname{disc}(V) \sim 1$ ), and also excluding the case $O_{j}(4,3,-1)$; or

- the reduction mod $p$ of one of the finite linear Coxeter groups of type $A_{n}(p \nmid n+1)$, $B_{n}, D_{n}, E_{6}(p \neq 3), E_{7}, E_{8}, F_{4}, H_{3}$ or $H_{4}$.

We shall say in these two cases that $G^{p}$ is of orthogonal or spherical type, respectively, although there is some overlap for small primes. (The notation $a \sim b$ means that $a=t^{2} b$ for some $t$ in the field.) Our description in [23, Thm. 3.1] rests on the classification of the finite irreducible reflection groups over any field, obtained in Zalesskiǔ \& Serežkin [37] (see also [17, 32, 33, 36]). For a non-singular space $V$, recall that $\epsilon=0$ if $n$ is odd, and $\epsilon=1$ or -1 if $n$ is even and the Witt index of $V$ is $n / 2$ or $(n / 2)-1$. When $n$ is even, $\operatorname{disc}(V) \sim(-1)^{n / 2}$ if $\epsilon=1$, and $\operatorname{disc}(V) \sim(-1)^{n / 2} \gamma$, with $\gamma$ a non-square, if $\epsilon=-1$. Moreover, $O_{1}(V)$ and $O_{2}(V)$ are the subgroups of $O(V)$ generated by the two distinct conjugacy classes of reflections, each characterized by the quadratic character of the spinor norm of its reflections, namely 1 or $\gamma$, respectively.

The above analysis sets up the stage for the construction of finite regular polytopes from crystallographic string Coxeter groups $G$. Clearly, the generators $r_{i}$ of the reduced group $G^{p}$ satisfy (at least) the Coxeter-type relations inherited from $G$. In most cases it also is more or less straightforward to determine the overall structure of $G^{p}$ by appealing to the 
above description of possible reflection groups. However, the main challenge is to determine when $G^{p}$ has the intersection property (2) for its standard subgroups. Here the outcome is often quite unpredictable, with the result depending on the underlying group $G$, the diagram $\Delta(G)$ used in the reduction, and the prime $p$. The reduced group $G^{p}$ may turn out to be a C-group for all primes, or for all primes in certain congruence classes, or for only finitely many primes (for example, only for $p=3$ ), or for no prime at all. Thus there is no theorem that covers all cases simultaneously.

Here we describe a quite general theorem (see [25, Thm. 2.3]) which, in the three basic cases considered, typically enables us to determine (often after additional considerations) whether or not the reduced group $G^{p}$ is a C-group. For any $k, l \in\{0, \ldots n-1\}$ we let $G_{k}^{p}:=\left\langle r_{j} \mid j \neq k\right\rangle$ and $G_{k, l}^{p}:=\left\langle r_{j} \mid j \neq k, l\right\rangle$. Then $G^{p}$ is a string C-group if and only if $G_{0}^{p}$ and $G_{n-1}^{p}$ are string C-groups and $G_{0}^{p} \cap G_{n-1}^{p}=G_{0, n-1}^{p}$. (This enables an inductive attack on the problem.) Similarly we let $V_{k}$ and $V_{k, l}$ denote the subspace of $V=\mathbb{Z}_{p}^{n}$ spanned by the vectors $b_{j}$ with $j \neq k$ or $j \neq k, l$, respectively. For a singular subspace $W$ of a non-singular space $V$, we also let $\widehat{O}(W)$ denote the subgroup of $O(W)$ consisting of those isometries which act trivially on the radical of $W$.

Theorem 3.1 Let $G=\left\langle r_{0}, \ldots, r_{n-1}\right\rangle$ be a crystallographic linear Coxeter group with string diagram. Suppose that $n \geq 3$, that the prime $p$ is generic for $G$ and that there is a square among the labels of the nodes $1, \ldots, n-2$ of the diagram $\Delta(G)$ (this can be achieved by readjusting the node labels). For various subspaces $W$ of $V$ we identify $O(W), \widehat{O}(W)$, etc. with suitable subgroups of the pointwise stabilizer of $W^{\perp}$ in $O(V)$.

(a) Let the subspaces $V_{0}, V_{n-1}$ and $V_{0, n-1}$ be non-singular, and let $G_{0}^{p}, G_{n-1}^{p}$ be of orthogonal type.

(i) Then $G_{0}^{p} \cap G_{n-1}^{p}$ acts trivially on $V_{0, n-1}^{\perp}$ and $O_{1}\left(V_{0, n-1}\right) \leq G_{0}^{p} \cap G_{n-1}^{p} \leq O\left(V_{0, n-1}\right)$.

(ii) If $G_{0}^{p}=O\left(V_{0}\right)$ and $G_{n-1}^{p}=O\left(V_{n-1}\right)$, then $G_{0}^{p} \cap G_{n-1}^{p}=O\left(V_{0, n-1}\right)$.

(iii) If either $G_{0}^{p}=O_{1}\left(V_{0}\right)$ or $G_{n-1}^{p}=O_{1}\left(V_{n-1}\right)$, then $G_{0}^{p} \cap G_{n-1}^{p}=O_{1}\left(V_{0, n-1}\right)$.

(b) Let $V, V_{0}, V_{n-1}$ be non-singular, let $V_{0, n-1}$ be singular (so that $n \geq 4$ ), and let $G_{0}^{p}, G_{n-1}^{p}$ be of orthogonal type.

(i) Then $G_{0}^{p} \cap G_{n-1}^{p}$ acts trivially on $V_{0, n-1}^{\perp}$, and $\widehat{O}_{1}\left(V_{0, n-1}\right) \leq G_{0}^{p} \cap G_{n-1}^{p} \leq \widehat{O}\left(V_{0, n-1}\right)$.

(ii) If $G_{0}^{p}=O\left(V_{0}\right)$ and $G_{n-1}^{p}=O\left(V_{n-1}\right)$, then $\widehat{O}\left(V_{0, n-1}\right)=G_{0}^{p} \cap G_{n-1}^{p}$.

(iii) If either $G_{0}^{p}=O_{1}\left(V_{0}\right)$ or $G_{n-1}^{p}=O_{1}\left(V_{n-1}\right)$, then $\widehat{O}_{1}\left(V_{0, n-1}\right)=G_{0}^{p} \cap G_{n-1}^{p}$.

(c) Suppose $V, V_{0, n-1}$ are non-singular while at least one of the subspaces $V_{0}, V_{n-1}$ is singular. Also suppose that $G_{0, n-1}^{p}$ is of orthogonal type, with $G^{p}=O_{1}(V)$ when $G_{0, n-1}^{p}=O_{1}\left(V_{0, n-1}\right)$. Then $G_{0}^{p} \cap G_{n-1}^{p}=G_{0, n-1}^{p}$.

We briefly discuss the modular polytopes associated with some interesting classes of crystallographic string Coxeter groups. Recall that $\left[p_{1}, p_{2}, \ldots, p_{n-1}\right]$ denotes the Coxeter group with a string Coxeter diagram on $n$ nodes and branches labelled $p_{1}, p_{2}, \ldots, p_{n-1}$. They are the automorphism groups of the universal regular polytopes $\left\{p_{1}, p_{2}, \ldots, p_{d-1}\right\}$ (see [21, §3D]).

\subsection{Groups in which $G$ has spherical or Euclidean Type}


The modular reduction $G^{p}$ of any spherical or euclidean (crystallographic) group $G$ is a string C-group for any prime $p \geq 3$, and $G^{p} \cong G$ if $G$ is spherical. There are four kinds of (connected) spherical string diagrams (up to duality), namely $A_{n}, B_{n}, F_{4}$ and $I_{2}(6)$ (dihedral of order 12). The corresponding modular polytopes are isomorphic to the $n$-simplex, $n$-cube, 24-cell and hexagon, respectively, and admit "modular realizations" in the finite space $\mathbb{Z}_{p}^{n}$. For the euclidean groups $\left[4,3^{n-3}, 4\right](n \geq 3),[3,4,3,3]$ and [ $\left.\infty\right]$, we obtain the regular toroids $\left\{4,3^{n-3}, 4\right\}_{\left(p, 0^{n-2}\right)}$ of rank $n$ and $\{3,4,3,3\}_{(p, 0,0,0)}$ of rank 5 , and the regular $p$-gon $\{p\}$.

For a spherical group $G$, we have $G^{d} \simeq G$ for any modulus $d \geq 3$, and sometimes even for $d=2$ (here depending on choice of diagram). For a euclidean group $G$ and $d \geq 3$ (again, sometimes for $d=2$ as well), the reduced group $G^{d}$ is the group of a regular toroid of rank $n$, but now its type vector (suffix) depends on $\Delta(G)$ (in particular, on the parity of $n$ ) and, as well, on the parity of $d$; the details are quite involved (see [26]).

\subsection{Groups of ranks 3 or 4}

The groups of ranks 1 or 2 are subsumed by our discussion in the previous paragraph. For rank 3, any group $G^{p}$ is a string C-group. For example, the hyperbolic group $G=[3, \infty]$ with diagram

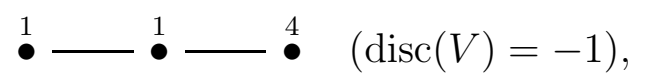

yields a regular map of type $\{3, p\}$. For $p \geq 5$ we find that $G^{p}=O_{1}(3, p, 0) \simeq P S L_{2}\left(\mathbb{Z}_{p}\right) \rtimes C_{2}$ of order $p\left(p^{2}-1\right)$. When $p=3,5$ or 7 , respectively, we obtain the regular tetrahedron $\{3,3\}$, icosahedron $\{3,5\}$ and the Klein polyhedron $\{3,7\}_{8}$. This construction redescribes the family of regular maps discussed (and generalized) in [19] or [20].

The situation changes drastically for groups $G$ of higher ranks, with obstructions already occurring in rank 4. We shall not attempt to fully summarize the findings of [24], which settle all groups $G=[k, l, m]$ of rank 4 (employing results such as Theorem 3.1). Suffice it here to mention some possible scenarios illustrating that the outcome is often unpredictable.

One possible scenario is that $G^{p}$ is a string C-group for all primes $p \geq 3$; this happens, for instance, if the subgroup $[k, l]$ or $[l, m]$ is spherical, if $l=\infty$, or if $[k, l]$ or $[l, m]$ is euclidean and $l=4$ or 6 . For example, from $[3,3, \infty]$ we obtain a regular 4 -polytope of type $\{3,3, p\}$ whose vertex-figures are isomorphic to the maps of type $\{3, p\}$ derived from $[3, \infty]$; its group $G^{p}$ is isomorphic to $S_{5}$ if $p=3, O_{1}(4,3,1)$ if $p \equiv 1 \bmod 4$, and $O_{1}(4,3,-1)$ if $p \equiv 3 \bmod 4$, $p \neq 3$. When $p=3,5$ or 7 , respectively, this gives the 4 -simplex $\{3,3,3\}$, the 600 -cell $\{3,3,5\}$, and the universal 4-polytope $\left\{\{3,3\},\{3,7\}_{8}\right\}$ first described in [27].

On the other extreme, there are groups $G$ for which $G^{p}$ is a C-group for only finitely many primes $p$. For example, for $G=[6,3,6]$ only $G^{3}$ is a C-group. More important examples arise when the four subspaces $V, V_{0}, V_{3}, V_{0,3}$ are all non-singular. For example, when $G=[\infty, 3, \infty]$ the reduced group $G^{p}$ is a C-group only for $p=3,5$ or 7 ; from $G^{3}$ and $G^{5}$ we obtain the 4 -simplex $\{3,3,3\}$ and the regular star-polytope $\left\{5,3, \frac{5}{2}\right\}$, and from $G^{7}$ a (non-universal) 4-polytope with facets and vertex-figures given by a dual pair of Klein maps $\{7,3\}_{8}$ and $\{3,7\}_{8}$.

Another possible scenario is that $G^{p}$ is a C-group only for certain congruence classes of primes. If $G=[6,3, \infty]$, then $G^{p}$ is a C-group if and only if $p=3$ or $p \equiv \pm 5 \bmod 12$.

\subsection{Groups of higher ranks}


The large number of crystallographic Coxeter groups $G$ of ranks $n \geq 5$ makes it difficult to fully enumerate the regular polytopes obtained by our method.

For the group $[4,3,4,3]$ of rank 5 we obtain regular 5 -polytopes with toroids $\{4,3,4\}_{(p, 0,0)}$ as facets and 24-cells $\{3,4,3\}$ as vertex-figures; their group $G^{p}$ is given by $O_{1}(5, p, 0)$ if $p \equiv \pm 1 \bmod 8$ and $O(5, p, 0)$ if $p \equiv \pm 3 \bmod 8$. These are examples of locally toroidal regular polytopes, meaning that their facets and vertex-figures are spherical or toroidal (but not all spherical). Such polytopes have not been fully classified (see [21, Ch.12]). The three closely related groups $[3,4,3,3,3],[3,3,4,3,3]$ and $[4,3,3,4,3]$ of rank 6 similarly give rise to locally toroidal 6 -polytopes with groups $O_{1}(6, p,+1)$ if $p \equiv \pm 1 \bmod 8$ and $O(6, p,+1)$ if $p \equiv \pm 3 \bmod 8$. Each polytope is covered by the respective universal polytope

$$
\begin{gathered}
\left\{\{3,4,3,3\}_{(p, 0,0,0)},\{4,3,3,3\}\right\}, \\
\left\{\{4,3,3,4\}_{(p, 0,0,0)},\{3,3,4,3\}_{(p, 0,0,0)}\right\}, \\
\left\{\{3,3,4,3\}_{(p, 0,0,0)},\{3,4,3,3\}_{(p, 0,0,0)}\right\},
\end{gathered}
$$

which has been conjectured to exist for all primes $p \geq 3$ and to be infinite for $p>3$ (see [21, Ch.12]). For $p=3$ (and $p=2$, but this is outside our discussion), these three universal polytopes are known to be finite. The first two have group $\mathbb{Z}_{3} \rtimes O(6,3,+1)$, and the last has group $\left(\mathbb{Z}_{3} \oplus \mathbb{Z}_{3}\right) \rtimes O(6,3,+1)$ (see $[25]$ ).

More generally we can reduce the four groups $G$ modulo any integer $d \geq 2$ to obtain other kinds of locally toroidal regular polytopes of ranks 5 or 6 , now with type vectors for facets and vertex-figures depending on $\Delta(G)$ and its subdiagrams for facets and vertex-figures, as well as on the parity of $d$ (see [26]). In particular, this establishes [21, Conjecture 12C2] concerning the existence of locally toroidal regular 6-polytopes of type $\{3,4,3,3,3\}$, saying that the universal regular 6-polytopes $\left\{\{3,4,3,3\}_{(d, 0,0,0)},\{4,3,3,3\}\right\}$ and $\left\{\{3,4,3,3\}_{(d, d, 0,0)},\{4,3,3,3\}\right\}$ exist for all $d \geq 2$; this then settles the existence of the first kind of 6-polytopes mentioned in the previous paragraph. The corresponding conjectures for the locally toroidal regular 6-polytopes of types $\{3,3,4,3,3\}$ and $\{4,3,3,4,3\}$ are still open (see [21, 12D3,12E3]).

Another interesting special class consists of the 3 -infinity groups $G=\left[p_{1}, \ldots, p_{n-1}\right]$, for which all periods $p_{j} \in\{3, \infty\}$. Typically then we have an alternating string of 3's and $\infty$ 's, and the outcome depends on the nature of the string. For the prime $p=3$ we always have a C-group, namely $S_{n+1}$. If $G=\left[3^{k}, \infty^{l}\right]$, with $k+l=n-1$, then for all primes $p \geq 3$, the group $G^{p}$ is a string $C$-group. We have seen examples with $n=3$ or 4 . For $G=\left[3, \infty^{l}, 3\right]$, with $n=l+3, l \geq 1$, we have a string $C$-group, except possibly when $p=7$ and $l \geq 4$, with $l \equiv 1 \bmod 3$. On the other hand, if $G=\left[3^{k}, \infty^{l}, 3^{m}\right]$, with $k>1$ or $m>1$, and $l \geq 1$, then $G^{p}$ is a string $C$-group for all but finitely many primes $p$. However, $G^{p}$ very often is not a C-group. For example, suppose that $G$ has a string subgroup of the form $\left[\ldots, \infty, 3^{k}, \infty, \ldots\right]$, with $k \geq 1$, and that $p \geq 5$; then $G^{p}$ is not a string $C$-group, except possibly when $p=5$ or 7 and $k \leq 1$ for all such string subgroups $\left[\ldots, \infty, 3^{k}, \infty, \ldots\right]$.

\section{Polytopes from hyperbolic Coxeter groups}

Following [31] we begin with the symmetry group $\Gamma=[r, s, t]$ of a regular honeycomb $\{r, s, t\}$ in hyperbolic 3-space $\mathbb{H}^{3}$ and consider a faithful representation of $\Gamma$ as a group of complex Möbius transformations. Recall here that the absolute of $\mathbb{H}^{3}$ can be identified with the complex inversive plane (compare the Poincare halfspace model of $\mathbb{H}^{3}$ ), and that every group of 
hyperbolic isometries is isomorphic to a group of Möbius transformations over $\mathbb{C}$. In particular, a plane reflection in $\mathbb{H}^{3}$ corresponds to an inversion in a circle (or line) determined as the "intersection" of the mirror plane in $\mathbb{H}^{3}$ with the absolute. Under this correspondence the generating plane reflections $\rho_{0}, \ldots, \rho_{3}$ of the hyperbolic reflection group $\Gamma$ become inversions in circles, again denoted by $\rho_{0}, \ldots, \rho_{3}$, cutting one another at the same angles as the corresponding reflection planes in $\mathbb{H}^{3}$. The group $\Gamma$ is one of ten possible groups, namely

$$
[4,4,3],[4,4,4],[6,3,3],[6,3,4],[6,3,5],[6,3,6],[3,6,3],[3,5,3],[5,3,4],[5,3,5] \text {; }
$$

see [34] for a complete list of the generating inversions for these groups.

Next recall that Möbius transformations may conveniently be represented (uniquely up to scalar multiplication) by $2 \times 2$ matrices, namely

$$
\frac{a z+b}{c z+d} \longleftrightarrow\left[\begin{array}{ll}
a & b \\
c & d
\end{array}\right] \quad \text { and } \quad \frac{a \bar{z}+b}{c \bar{z}+d} \longleftrightarrow{ }^{\#}\left[\begin{array}{ll}
a & b \\
c & d
\end{array}\right]
$$

with appropriate interpretations for multiplication of such matrices. We then obtain matrices $r_{0}, r_{1}, r_{2}, r_{3}$ and $s_{1}, s_{2}, s_{3}$, respectively, for the generators $\rho_{0}, \rho_{1}, \rho_{2}, \rho_{3}$ of $\Gamma$ and $\sigma_{1}, \sigma_{2}, \sigma_{3}$ of $\Gamma^{+}$. Let $G$ denote the group of complex $2 \times 2$ matrices generated by $s_{1}, s_{2}, s_{3}$. When considered modulo scalars, $G$ (or rather the corresponding projective group $P G$ ) is isomorphic to $\Gamma^{+}$, the rotation group of $\{r, s, t\}$. It turns out that the matrices in $G$ all have entries in a certain subring $\mathbb{D}$ of $\mathbb{C}$ depending on $[r, s, t]$. Thus modular reduction applies. We choose appropriate ideals $J$ in $\mathbb{D}$ and consider the matrices $s_{1}, s_{2}, s_{3}$ over the quotient ring $\mathbb{D} / J$, again modulo scalars (determined by a suitable subgroup of the center). Then under certain conditions, the resulting projective group $G^{J}$ (say) is either the rotation subgroup for a directly regular polytope, or the full automorphism group of a chiral polytope.

We illustrate the method for the hyperbolic group $[4,4,3]$. When $\Gamma=[4,4,3]$ is viewed as a group of complex Möbius transformations, the generators may be taken as

$$
\rho_{0}(z)=\bar{z}, \quad \rho_{1}(z)=i \bar{z}, \quad \rho_{2}(z)=1-\bar{z}, \quad \rho_{3}(z)=1 / \bar{z} .
$$

Then the rotation subgroup $\Gamma^{+}$of $\Gamma$ is generated by

$$
\sigma_{1}=\rho_{0} \rho_{1}=-i z, \quad \sigma_{2}=\rho_{1} \rho_{2}=-i z+i, \quad \sigma_{3}=\rho_{2} \rho_{3}=1-1 / z,
$$

and consists only of proper Möbius transformations (not involving complex conjugation). Here the matrices $s_{1}, s_{2}, s_{3}$ for $\sigma_{1}, \sigma_{2}, \sigma_{3}$ are given by

$$
s_{1}=\left[\begin{array}{rr}
-i & 0 \\
0 & 1
\end{array}\right], \quad s_{2}=\left[\begin{array}{rr}
-i & i \\
0 & 1
\end{array}\right], \quad s_{3}=\left[\begin{array}{rr}
1 & -1 \\
1 & 0
\end{array}\right],
$$

and $G$ is the group of all invertible $2 \times 2$ matrices over the Gaussian integers $\mathbb{D}=\mathbb{Z}[i]$ (with determinants $\pm 1, \pm i)$. In particular, $[4,4,3]^{+} \cong P S L_{2}(\mathbb{Z}[i]) \rtimes C_{2}$, where the first factor is also known as the Gaussian modular group or Picard group (see also [15]). Since we wish to obtain toroidal facets of type $\{4,4\}_{(b, c)}^{+}$, we must consider the imposition of the extra relation $\left(\sigma_{1}^{-1} \sigma_{2}\right)^{b}\left(\sigma_{1} \sigma_{2}^{-1}\right)^{c}=1$ on the generators of $\Gamma^{+}$(see [8]). Note here that the corresponding matrix product in $G$ is

$$
\left(s_{1}^{-1} s_{2}\right)^{b}\left(s_{1} s_{2}^{-1}\right)^{c}=\left[\begin{array}{rr}
1 & -1 \\
0 & 1
\end{array}\right]^{b}\left[\begin{array}{rr}
1 & -i \\
0 & 1
\end{array}\right]^{c}=\left[\begin{array}{cc}
1 & -(b+c i) \\
0 & 1
\end{array}\right] .
$$


Thus the ideals $J$ of $\mathbb{Z}[i]$ must be chosen in such a way that $b+c i=0$ in $\mathbb{Z}[i] / J$.

One natural choice of ideal is $J=m \mathbb{Z}[i]$, where $m \geq 3$ is an integer in $\mathbb{Z}$. This choice of ideal typically produces directly regular polytopes of type $\left\{\{4,4\}_{(m, 0)},\{4,3\}\right\}$ whose rotation subgroup is $P S L_{2}\left(\mathbb{Z}_{m}[i]\right)$ or a closely related group, with the exact structure determined by the prime factorization of $m$ in $\mathbb{Z}$ (see [31, p.238]). For example, if $m \equiv 3 \bmod 4$ is a prime, we obtain $P S L_{2}\left(\mathbb{Z}_{m}[i]\right) \cong P S L_{2}\left(m^{2}\right)$.

A more interesting choice of ideal arises from a solution of the equation $x^{2}=-1 \bmod m$, with $m$ as above. Let $m=2^{e} p_{1}^{e_{1}} \ldots p_{k}^{e_{k}}$ be the prime factorization in $\mathbb{Z}$. Then the equation is solvable if and only if $e=0,1$ and $p_{j} \equiv 1 \bmod 4$ for each $j$. If $\hat{i} \in \mathbb{Z}_{m}$ is such that $\hat{i}^{2}=-1 \bmod m$, then there exists a unique pair of positive integers $b, c$ such that $m=b^{2}+c^{2}$, $(b, c)=1$ and $b=-\hat{i} c \bmod m$. We now take $J=(b+c i) \mathbb{Z}[i]$, which is the kernel of the ring epimorphism $\mathbb{Z}[i] \rightarrow \mathbb{Z}_{m}$ that maps the complex number $x+y i$ to the element $x_{m}+y_{m} \hat{i}$ in $\mathbb{Z}_{m}$, where $x_{m} \equiv x$ and $y_{m} \equiv y \bmod m$. This choice of ideal "destroys" the reflections in the overlying reflection group and typically yields chiral polytopes of type $\left\{\{4,4\}_{(b, c)},\{4,3\}\right\}$ whose rotation subgroup is $P S L_{2}\left(\mathbb{Z}_{m}\right)$ or a closely related group, with the exact structure again determined by the prime factorization of $m$. For example, if $m \equiv 1 \bmod 8$ is a prime, then the group is $P S L_{2}(m)$. Note that the structure of the polytope also depends on the solution $\hat{i}$. For example, when $m=65$, the solution $\hat{i}=8$ gives facets $\{4,4\}_{(1,8)}$, while $\hat{i}=18$ leads to facets $\{4,4\}(4,7)$.

With similar techniques we can construct a host of regular or chiral 4-polytopes of types $\{4,4,4\},\{4,4,4\},\{6,3,3\},\{6,3,4\},\{6,3,5\},\{6,3,6\}$ or $\{3,6,3\}$ (see [30, 31]). However, the underlying ring $\mathbb{D}$ will depend on the Schläfli symbol. The Gaussian integers $\mathbb{Z}[i]$ and Eisenstein integers $\mathbb{Z}[\omega]$ (with $\omega$ a cube root of unity) suffice in all cases except $\{6,3,4\}$ and $\{6,3,5\}$ (this is based on subgroup relationships between the rotation subgroups for the various types). For $\{6,3,4\}$ and $\{6,3,5\}$, respectively, we can work over the ring $\mathbb{Z}[\omega, \sqrt{2}]$ and $\mathbb{Z}[\omega, \tau]$ (with $\tau$ the golden ratio; see the next section).

Chiral polytopes also exist in ranks larger than 4, but explicit constructions of finite examples (or rank 5) were only discovered quite recently in [4]. Modular reduction is an effective method to produce examples in rank 4. In addition to the symmetry groups of 3-dimensional hyperbolic honeycombs, several other discrete hyperbolic groups admit representations as groups of linear fractional transformations over other rings of complex or quaternionic integers (described in detail in the forthcoming book by Johnson [15]). Although the arithmetic involved is likely to be considerably more complicated than in the rank 4 case, there is a good chance that the reduction method will carry over to produce examples of finite chiral polytopes of rank larger than 4.

\section{The groups $[3,5,3]$ and $[5,3,5]$}

In Section 3 we observed that any crystallographic string Coxeter group $G$ has rotational periods $p_{j} \in\{2,3,4,6, \infty\}$ and can be represented faithfully as a matrix group over the domain $\mathbb{Z}$. Here we widen the discussion a little by allowing the period $p_{j}=5$. Keeping

(44) in mind, we note that $2 \cos \frac{\pi}{5}=\tau$, where the golden ratio $\tau=(1+\sqrt{5}) / 2$ is the positive 
root of $\tau^{2}=\tau+1$. We therefore move to the larger coefficient domain

$$
\mathbb{D}:=\mathbb{Z}[\tau]=\{a+b \tau: a, b \in \mathbb{Z}\}
$$

and soon find that we need only add the subdiagram

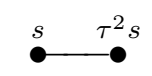

to those already listed in Table 1 in order for the Cartan integers $m_{i j}$ of (5) to be in $\mathbb{D}$ for all $i, j$. This subdiagram, say on nodes $i, j$, does indeed define the non-crystallographic dihedral group $\left\langle r_{i}, r_{j}\right\rangle$ with order 10 and period $p_{i j}=5$. (In other notation, this is the group $H_{2} \simeq I_{2}(5)$.) Naturally, we must now allow rescaling of nodes by any 'integer' $s \in \mathbb{D}$ or its inverse. Furthermore, referring back to (5), we find that $m_{i j}=\tau^{2} \in \mathbb{D}$, so that $G$ is represented as a matrix group over $\mathbb{D}$ through its action on the $\mathbb{D}$-module $\oplus_{j} \mathbb{D} b_{j}$.

Let us now summarize the key arithmetic properties of the domain $\mathbb{D}$. (We refer to [10] for a detailed account of this, and to [3] for a deeper discussion of ' $\mathbb{D}$-lattices' and the related finite Coxeter groups $H_{k}, k=2,3,4$.) First of all, we recall that $\mathbb{D}$ is the ring of algebraic integers in the field $\mathbb{Q}(\sqrt{5})$. The non-trivial field automorphism mapping $\sqrt{5} \mapsto-\sqrt{5}$ induces a ring automorphism ' $: \mathbb{D} \rightarrow \mathbb{D}$, which in this section we shall call conjugation. Thus

$$
(a+b \tau)^{\prime}=(a+b)-b \tau .
$$

In particular, $\tau^{\prime}=1-\tau=-\tau^{-1}$. Recall that $z=a+b \tau$ has norm $N(z):=z z^{\prime}=a^{2}+a b-b^{2}$. We note that $\mathbb{D}$ is a Euclidean domain, through a division algorithm based on $|N(z)|$.

The set of units in $\mathbb{D}$ is $\left\{ \pm \tau^{n}: n \in \mathbb{Z}\right\}=\{u \in \mathbb{D}: N(u)= \pm 1\}$. Recall that integers $z, w \in \mathbb{D}$ are associates if $z=u w$ for some unit $u$. Up to associates, the primes $\pi \in \mathbb{D}$ can be classified as follows:

- the prime $\pi=\sqrt{5}=2 \tau-1$, which is self-conjugate (up to associates: $\pi^{\prime}=-\pi$ );

- rational primes $\pi=p \equiv \pm 2 \bmod 5$, also self-conjugate;

- primes $\pi=a+b \tau$, for which $|N(\pi)|$ equals a rational prime $q \equiv \pm 1 \bmod 5$. In this case, the conjugate prime $\pi^{\prime}=(a+b)-b \tau$ is not an associate of $\pi$.

Let us now turn to the group $G=[3,5,3]$, here acting as an orthogonal group on real 4-space $V$ (in contrast to the conformal representation on $\mathbb{H}^{3}$ mentioned in the previous section). Since $\tau^{2}$ is a unit, there is essentially only one choice of diagram, namely

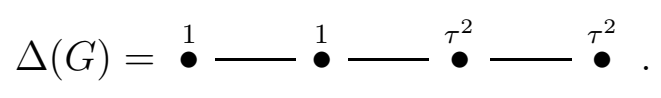

The discriminant is

$$
\operatorname{disc}(V)=-\frac{1}{16}(2+5 \tau) \sim-(2+5 \tau)
$$

where the prime $\delta:=-(2+5 \tau)$ has norm -11 .

Now consider any prime $\pi \in \mathbb{D}$. Our goals are to show that $G^{\pi}=\left\langle r_{0}, r_{1}, r_{2}, r_{3}\right\rangle^{\pi}$ is a string $C$-group and to determine its structure, then say a little about the corresponding polytope $\mathcal{P}^{\pi}:=\mathcal{P}\left(G^{\pi}\right)$. 
In fact, we can almost immediately apply a suitable generalization of Theorem 4.2 in [23]. First note that the subgroup $G_{3}^{\pi}=\left\langle r_{0}, r_{1}, r_{2}\right\rangle^{\pi}$ is obviously some quotient of the spherical group $[3,5] \simeq H_{3}$. Now it is easy to check that after reduction modulo any prime $\pi$, even for associates of 2 , the reflections $r_{j}$ still have period 2. Next, we consider the isometry

$$
z:=\left(r_{0} r_{1} r_{2}\right)^{5}=\left[\begin{array}{cccc}
-1 & 0 & 0 & \tau^{4} \\
0 & -1 & 0 & 2 \tau^{4} \\
0 & 0 & -1 & 3 \tau^{2} \\
0 & 0 & 0 & 1
\end{array}\right]
$$

in $G$. Since $\tau^{4}$ is a unit, this means that $r_{0} r_{1} r_{2}$ still has period $10 \mathrm{in} G^{\pi}$. Thus $\left\langle r_{0}, r_{1}, r_{2}\right\rangle^{\pi} \simeq$ $[3,5]$ and dually $\left\langle r_{1}, r_{2}, r_{3}\right\rangle^{\pi} \simeq[5,3]$. Consulting the proof of [23, Th. 4.2], we see that we need only show that the orbit of $\mu_{0}:=[1,0,0,0]$ under the right action of the matrix group $\left\langle r_{0}, r_{1}, r_{2}\right\rangle$ has the same size modulo $\pi$ as in characteristic 0 , namely 12 . This is routinely verified, so we have proved most of

Proposition 5.1 Let $G=[3,5,3]$. For any prime $\pi \in \mathbb{D}$, the group $G^{\pi}=\left\langle r_{0}, r_{1}, r_{2}, r_{3}\right\rangle^{\pi}$ is a finite string $C$-group. The corresponding finite regular polytope $\mathcal{P}^{\pi}$ is self-dual and has icosahedral facets $\{3,5\}$ and dodecahedral vertex figures $\{5,3\}$.

Proof. To verify self-duality we define $g \in G L(V)$ by $g:\left[b_{0}, b_{1}, b_{2}, b_{3}\right] \mapsto\left[\tau^{-1} b_{3}, \tau^{-1} b_{2}, \tau b_{1}, \tau b_{0}\right]$. Then $g^{2}=1, g r_{0} g=r_{3}$ and $g r_{1} g=r_{2}$. (See [21, 2E12].)

A more detailed description of $G^{\pi}$ must depend on the nature of the prime $\pi$. (Of course, our results are typically unaffected by replacing $\pi$ by any associate $\pm \tau^{m} \pi$.) In all cases the underlying finite field $\mathbb{K}:=\mathbb{D} /(\pi)$ has order $|N(\pi)|$, so that $G^{\pi}$ acts as an orthogonal group on the 4-dimensional vector space $V$ over $\mathbb{K}$ preserving the modular image of the bilinear form for $G$.

Case 1: $\pi=2$.

Here an easy calculation using GAP confirms that $G^{\pi}$ is the orthogonal group $O\left(4,2^{2},-1\right)$ with Witt index 1 over $\mathbb{K}=G F\left(2^{2}\right)$. Since $\left|G^{2}\right|=8160$, the polytope $\mathcal{P}^{2}$ has 68 vertices and 68 icosahedral facets.

Henceforth we suppose that $\pi$ is not an associate of 2 . To work with such primes, we need a generalization of the rational Legendre symbol $(p \mid q)$. Thus for any $\alpha \in \mathbb{D}$ and prime $\pi$ we set

$$
(\alpha \mid \pi)_{\mathbb{D}}:= \begin{cases}+1, & \text { if } \alpha \text { is a quadratic residue }(\bmod \pi) \\ -1, & \text { otherwise. }\end{cases}
$$

(Compare [10, Ch. VIII].) We are mainly interested in computing

$$
\epsilon:=(\delta \mid \pi)_{\mathbb{D}}
$$

where $\delta=-(2+5 \tau)$ is the discriminant. Since every label in $\Delta(G)$ is square, we conclude that $G^{\pi}$ is a subgroup of $O_{1}(4,|N(\pi)|, \epsilon)$, so long as $\delta$ and $\pi$ are relatively prime. Indeed, $G^{\pi}$ will almost always equal such an orthogonal group. 
Case 2: $\pi=\sqrt{5}=2 \tau-1$.

Here $\left|\pi \pi^{\prime}\right|=5 \equiv 0 \bmod \pi$, so that the discriminant $\delta=-(2+5 \tau) \equiv 3 \bmod \pi$, which is non-square in $\mathbb{K}=G F(5)$. Thus $\epsilon=-1$ and $G^{\pi}=O_{1}(4,5,-1)$ has order 15600. In fact, the polytope $\mathcal{P}^{\sqrt{5}}$ is isomorphic to that obtained in [24, p. 347] through reduction mod5 of the crystallographic group $[3, \infty, 3]$.

Case 3: $\pi$ is an associate of an odd rational prime $p \equiv \pm 2 \bmod 5$.

Since $\mathbb{K}=G F\left(p^{2}\right), G^{\pi}=G^{p}$ is, for suitable $\epsilon$, a subgroup of $O_{1}\left(4, p^{2}, \epsilon\right)$, whose order we recall is $p^{4}\left(p^{4}-\epsilon\right)\left(p^{4}-1\right)$. Consulting [23, Th. 3.1], we see that $G^{p}=O_{1}\left(4, p^{2}, \epsilon\right)$ so long as we can rule out two remote alternatives.

First of all, it is conceivable that $G^{p} \simeq H_{4}=[3,3,5]$. But here it is easy to check directly that $H_{4}$ cannot be generated by reflections $r_{j}$ satisfying the Coxeter-type relations inherited from $[3,5,3]$, let alone the independent relations induced by reduction modulo $p$.

Secondly, we must show that $G^{p}$ is not isomorphic to some orthogonal group $O_{1}(4, p, \eta)$, $\eta= \pm 1$, over the subfield $G F(p)$. If this were so, then Theorem 3.1 in [23] would actually imply that $G^{p}$ is similar to $O_{1}(4, p, \eta)$ under extension of scalars. More precisely, if $\mathbb{L}$ is an algebraic closure of $\mathbb{K}=G F\left(p^{2}\right)$, then there would exist some $g \in G L\left(V_{\mathbb{L}}\right)$ with $g G^{p} g^{-1}=$ $O_{1}(4, p, \eta)$. Using (5) , we compute with respect to the new basis $\left\{c_{i}\right\}=\left\{g\left(b_{i}\right)\right\}$ for $V_{\mathbb{L}}$. Thus the reflection $\tilde{r}_{i}:=g r_{i} g^{-1}$ satisfies

$$
\tilde{r}_{i}\left(c_{j}\right)=g\left(b_{j}+m_{i j} b_{i}\right)=c_{j}+m_{i j} c_{i}
$$

We conclude that the field of definition for $G^{p}$ must always contain the subfield generated by the Cartan integers $m_{i j}$. In our case, $m_{12}=\tau^{2} \notin G F(p)$, so that $g G^{p} g^{-1}$ cannot possibly be a group $O_{1}(4, p, \eta)$.

Having shown that $G^{p}=O_{1}\left(4, p^{2}, \epsilon\right)$, we next determine $\epsilon$. From [10, Th. 8.5(a)] we have

$$
(\delta \mid \pi)_{\mathbb{D}}=(N(\delta) \mid p)=(-11 \mid p)=(-1 \mid p)(11 \mid p)=(p \mid 11),
$$

by (rational) quadratic reciprocity. Since the non-zero squares $(\bmod 11)$ are $1,3,4,5,9$, we conclude that

$$
\epsilon:= \begin{cases}+1, & \text { if } p \equiv 3,12,23,27,37,38,42,47,48,53 \bmod 55 \\ -1, & \text { if } p \equiv 2,7,8,13,17,18,28,32,43,52 \bmod 55\end{cases}
$$

Case 4: $\pi=a+b \tau$, where $N(\pi)=a^{2}+a b-b^{2}=q$, where the rational prime $q \equiv \pm 1 \bmod 5$; however, $\pi$ is not an associate of $\delta=-(2+5 \tau)$.

We now have $\mathbb{K}=G F(q)$. An even easier appeal to [23, Th. 3.1] gives $G^{\pi}=O_{1}(4, q, \epsilon)$. We need only determine $\epsilon=(\delta \mid \pi)_{\mathbb{D}}$. Since $a+b \tau \equiv 0 \bmod \pi$, we may suppose $\tau=-a / b \in$ $\mathbb{K}$. Thus

$$
\delta=-(2+5 \tau) \sim-b^{2}(2+5 \tau) \equiv 5 a b-2 b^{2} \bmod \pi
$$

By [10, Th. 8.5(a)], we obtain

$$
\epsilon=(\delta \mid \pi)_{\mathbb{D}}=\left(\left(5 a b-2 b^{2}\right) \mid q\right)=(b \mid q)((5 a-2 b) \mid q) .
$$

Using the rational Legendre symbol, we can thus compute $\epsilon$ for any prime $\pi=a+b \tau$. 
It is possible to say when $\pi$ and its conjugate $\pi^{\prime}$ give opposite $\epsilon$ 's, so that the corresponding orthogonal spaces have, in some order, Witt indices 1 and 2. This happens if and only if $q$ is a square mod 11 , since

$$
(\delta \mid \pi)_{\mathbb{D}}\left(\delta \mid \pi^{\prime}\right)_{\mathbb{D}}=((b(5 a-2 b)(-b)(5 a+7 b)) \mid q)=(-11 \mid q)=(q \mid 11) .
$$

One notable instance here is $\pi=\delta^{\prime}=-7+5 \tau$, which is relatively prime to the discriminant $\delta$. We have $G^{\delta^{\prime}}=O_{1}(4,11,-1)$, of order 1771440 .

Case 5: $\pi=\delta=-(2+5 \tau)$.

This is the only case in which the orthogonal space $V$ is singular. Now $\mathbb{K}=G F(11)$ and $\tau=-2 / 5=4$. We find that $\operatorname{rad}(V)$ is spanned by $c=7 b_{0}+3 b_{1}+2 b_{2}+b_{3}$, and that $V=\operatorname{rad}(V) \perp V_{3}$, where $V_{3}$ is the non-singular subspace spanned by $b_{0}, b_{1}, b_{2}$. It is then not hard to see that

$$
O(V) \simeq \check{V}_{3} \rtimes\left(\mathbb{K}^{*} \times O\left(V_{3}\right)\right)
$$

where $\mathbb{K}^{*} \simeq G L(\operatorname{rad}(V))$ and $\check{V}_{3}$ is dual to $V_{3}$. We observe that the abelian group $\check{V}_{3} \simeq \mathbb{K}^{3}$ consists of all transvections

$$
r(x)=x+\varphi(x) c
$$

where $\varphi \in \check{V}_{3}$ (with $\check{V}_{3}$ viewed as a subspace of $\check{V}$ fixing $c$ ). Now since every $r_{j}$ fixes $c, G^{\delta}$ must be a subgroup of the pointwise stabilizer of $\operatorname{rad}(V)$. In fact, another calculation with GAP confirms that

$$
G^{\delta}=\widehat{O}_{1}(V) \simeq \check{V}_{3} \rtimes O_{1}\left(V_{3}\right),
$$

which has order $11^{3} \cdot 11 \cdot\left(11^{2}-1\right)=1756920$. Now consider the isometry $z \in G$ defined in (6) $)$. It is easy to check that $z(c) \equiv c \bmod \delta$, so that $z=1_{\operatorname{rad}(\mathrm{V})} \perp-1_{V_{3}} \in \widehat{O}_{1}(V)$ acts as the central inversion in the group $O_{1}\left(V_{3}\right)$ for the icosahedral facet. Thus $G^{\delta}$ has a normal subgroup $A$ isomorphic to $\check{V}_{3} \rtimes\langle z\rangle$ and so of order $2 \cdot 11^{3}$. Using $O_{1}(3,11,0) \simeq P S L_{2}(11) \rtimes C_{2}$ (see [1, Th. 5.20]), we conclude that

$$
\bar{G}:=G^{\delta} / A \simeq P S L_{2}(11),
$$

of order 660. Remarkably, $\bar{G}$ is also a string $C$-group. The resulting polytope is the 11-cell independently discovered by Coxeter in [7] and Grünbaum in [11]. Indeed, both $r_{0} r_{1} r_{2}$ and $r_{1} r_{2} r_{3}$ have period 5 in the quotient (see (6) ), and

$$
\mathcal{P}(\bar{G})=\left\{\{3,5\}_{5},\{5,3\}_{5}\right\}
$$

is the universal 4-polytope with hemi-icosahedral facets and hemi-dodecahedral vertexfigures.

This finishes our investigation of the group $[3,5,3]$. Evidently a somewhat similar analysis is possible for the group $H=[5,3,5]$ with diagram

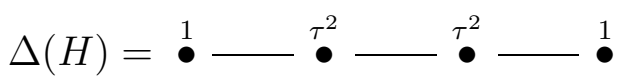

and corresponding discriminant $\frac{-1}{16}(3+7 \tau) \sim-(3+7 \tau)=$ : $\lambda$. Since $N(\lambda)=-19$, we see that $\lambda$ is also prime. We note only that the group $H^{\lambda}$ for the singular space $V$ again has an interesting quotient. In fact,

$$
\bar{H} \simeq P S L_{2}(19)
$$


is the automorphism group for the universal regular polytope

$$
\mathcal{P}(\bar{H})=\left\{\{5,3\}_{5},\{3,5\}_{5}\right\}
$$

with hemi-dodecahedral facets and hemi-icosahedral vertex-figures. This is the 57-cell described by Coxeter in [6].

With the exception of the 11-cell and 57-cell, the polytopes described here can be viewed as regular tessellations on hyperbolic 3-manifolds (see [21, 6J]). Moreover, the two exceptions are the only regular polytopes or rank 4 (or higher) with automorphism group isomorphic to $P S L_{2}(r)$ for some prime power $r$ (see [18]). For related work see also [12, 13, 16].

\section{References}

[1] E. Artin, Geometric Algebra, Interscience, New York (1957).

[2] T. Bisztriczky, P. McMullen, R. Schneider and A. Ivić Weiss (Eds.), Polytopes Abstract, Convex and Computational, NATO ASI Series C440 (Kluwer, 1994).

[3] L. Chen, R. Moody and J. Patera, Non-crystallographic root systems, QuAsicrystals AND DisCRETE GeOMETRY, Fields Inst. Monogr. 10 (AMS, 1998), pp. 135-178.

[4] M. Conder, I. Hubard and T. Pisanski, Constructions for chiral polytopes, Journal London Mathematical Society (to appear).

[5] H.S.M. Coxeter, Extreme forms, Canadian J. Math., 3 (1951), 391-441.

[6] H.S.M. Coxeter, Ten toroids and fifty-seven hemi-dodecahedra, Geom. Dedicata, 13 (1982), 87-99.

[7] H.S.M. Coxeter, A symmetrical arrangement of eleven hemi-icosahedra. In ConvEXITY and Graph Theory (Jerusalem, 1981), North-Holland Math. Stud. 87, NorthHolland (Amsterdam, 1984), pp. 391-441.

[8] H.S.M. Coxeter and W.O.J. Moser, Generators and Relations for Discrete Groups, 3Rd Ed., Springer, New York (1972).

[9] C.W. Curtis and I. Reiner, Representation Theory of Finite Groups and Associative Algebras, Wiley-Interscience, New York (1962).

[10] F.W. Dodd, Number Theory in the Quadratic Field with Golden Section UnIt, Polygonal Publishing House, Passaic, NJ (1983).

[11] B. Grünbaum, Regularity of graphs, complexes and designs. In Problèmes combinatoires et théorie des graphes, Colloq. Internat. C.N.R.S. No. 260, Orsay (1977), pp. 191-197.

[12] M.I. Hartley and D. Leemans, Quotients of a universal locally projective polytope of type $\{5,3,5\}$, Math. Zeitschrift 247 (2004), 663-674.

[13] M.I. Hartley and D. Leemans, On locally spherical polytopes of type $\{5,3,5\}$, Discrete Math. (2008, to appear).

[14] J.E. Humphries, Reflection Groups and Coxeter Groups, Cambridge University Press, Cambridge, 1990.

[15] N. Johnson, Geometries and Transformations, book manuscript.

[16] G.A. Jones and C.D. Long, Epimorphic images of the [5,3,5] Coxeter group, Preprint.

[17] W. Kantor, Generation of linear groups, The Geometric Vein - The Coxeter Festschrift, Springer, New York-Berlin, 1981, pp. 497-509. 
[18] D. Leemans and E. Schulte, Groups of type $L_{2}(q)$ acting on polytopes, Advances in Geometry 7 (2007), 529-539.

[19] P. McMullen, Regular polyhedra related to projective linear groups, Discrete Mathematics. 91 (1991), 161-170.

[20] P. McMullen, B.R. Monson and A.I. Weiss, Regular maps constructed from linear groups, European J. Combin. 14 (1993), 541-552.

[21] P. McMullen and E. Schulte, Abstract Regular Polytopes, Encyclopedia of Mathematics and its Applications, 92, Cambridge University Press, Cambridge, 2002.

[22] B. Monson, Simplicial Quadratic Forms, Canad. J. Math. 35 (1983), 101-116.

[23] B. Monson and E. Schulte, Reflection groups and polytopes over finite fields, I, Adv. in Appl. Math. 33 (2004), 290-317.

[24] B. Monson and E. Schulte, Reflection groups and polytopes over finite fields, II, Adv. in Appl. Math. 38 (2007), 327-356.

[25] B. Monson and E. Schulte, Reflection groups and polytopes over finite fields, III, Adv. in Appl. Math., (to appear).

[26] B. Monson and E. Schulte, Locally toroidal polytopes and modular linear groups, Preprint.

[27] B. Monson and A.I. Weiss, Regular 4-polytopes related to general orthogonal groups, Mathematika 37 (1990), 106-118.

[28] B. Monson and A.I. Weiss, Polytopes related to the Picard Group, Linear Algebra Appl. 218 (1995), 185-204.

[29] B. Monson and A.I. Weiss, Eisenstein Integers and Related C-Groups, Geom. Dedicata 66 (1997), 99-117.

[30] B. Nostrand and E. Schulte, Chiral polytopes from hyperbolic honeycombs, Discrete Comput. Geom. 13 (1995), 17-39.

[31] E. Schulte and A.I. Weiss, Chirality and projective linear groups, Discrete. Math. 131 (1994), 221-261.

[32] A. Wagner, Determination of the finite primitive reflection groups over an arbitrary field of characteristic not two. I, Geom. Dedicata 9 (1980), 239-253.

[33] A. Wagner, Determination of the finite primitive reflection groups over an arbitrary field of characteristic not two. II, III, Geom. Dedicata 10 (1981), 191-203 and 475-523.

[34] A.I. Weiss, Incidence polytopes with toroidal cells, Discrete \& Computational Geometry 4 (1989), 55-73.

[35] S.E. Wilson, Applications and refinements of Vince's construction, Geom. Dedicata 48 (1993), 231-242.

[36] A.E. Zalesskiu and V.N. Serežkin, Linear groups generated by transvections. (Russian) Izv. Akad. Nauk SSSR Ser. Mat. 40 (1976), 26-49. (English translation in Math. USSR Izvestija 10 (1976), 25-46.)

[37] A.E. Zalesskiı̌ and V.N. Serežkin, Finite linear groups generated by reflections. (Russian) Izv. Akad. Nauk SSSR Ser. Mat. 44 (1980), 1279-1307, 38. (English translation in Math. USSR Izvestija 17 (1981), 477-503.)

B. Monson, University of New Brunswick, Fredericton, New Brunswick, Canada E3B 5A3, bmonson@unb.ca

Egon Schulte, Northeastern University, Boston, Massachussetts, USA, 02115, schulte@neu.edu 